\title{
Prospective, multidisciplinary recording of perioperative errors in cerebrovascular surgery: is error in the eye of the beholder?
}

\author{
Suzanne M. Michalak, ScB, ${ }^{1}$ John D. Rolston, MD, PhD, ${ }^{2}$ and Michael T. Lawton, MD² \\ ${ }^{1}$ School of Medicine, University of North Carolina, Chapel Hill, North Carolina; and 2Department of Neurological Surgery, \\ University of California, San Francisco, California
}

\begin{abstract}
OBJECTIVE Surgery requires careful coordination of multiple team members, each playing a vital role in mitigating errors. Previous studies have focused on eliciting errors from only the attending surgeon, likely missing events observed by other team members.
\end{abstract}

METHODS Surveys were administered to the attending surgeon, resident surgeon, anesthesiologist, and nursing staff immediately following each of 31 cerebrovascular surgeries; participants were instructed to record any deviation from optimal course (DOC). DOCs were categorized and sorted by reporter and perioperative timing, then correlated with delays and outcome measures.

RESULTS Errors were recorded in $93.5 \%$ of the 31 cases surveyed. The number of errors recorded per case ranged from 0 to 8 , with an average of $3.1 \pm 2.1$ errors ( \pm SD). Overall, technical errors were most common $(24.5 \%)$, followed by communication (22.4\%), management/judgment (16.0\%), and equipment $(11.7 \%)$. The resident surgeon reported the most errors $(52.1 \%)$, followed by the circulating nurse $(31.9 \%)$, the attending surgeon $(26.6 \%)$, and the anesthesiologist $(14.9 \%)$. The attending and resident surgeons were most likely to report technical errors ( $52 \%$ and $30.6 \%$, respectively), while anesthesiologists and circulating nurses mostly reported anesthesia errors (36\%) and communication errors (50\%), respectively. The overlap in reported errors was $20.3 \%$. If this study had used only the surveys completed by the attending surgeon, as in prior studies, $72 \%$ of equipment errors, $90 \%$ of anesthesia and communication errors, and $100 \%$ of nursing errors would have been missed. In addition, it would have been concluded that errors occurred in only $45.2 \%$ of cases (rather than $93.5 \%$ ) and that errors resulting in a delay occurred in $3.2 \%$ of cases instead of the $74.2 \%$ calculated using data from 4 team members. Compiled results from all team members yielded significant correlations between technical DOCs and prolonged hospital stays and reported and actual delays ( $p=0.001$ and $p=0.028$, respectively).

CONCLUSIONS This study is the only of its kind to elicit error reporting from multiple members of the operating team, and it demonstrates error is truly in the eye of the beholder-the types and timing of perioperative errors vary based on whom you ask. The authors estimate that previous studies surveying only the attending physician missed up to $75 \%$ of perioperative errors. By finding significant correlations between technical DOCs and prolonged hospital stays and reported and actual delays, this study shows that these surveys provide relevant and useful information for improving clinical practice. Overall, the results of this study emphasize that research on medical error must include input from all members of the operating team; it is only by understanding every perspective that surgical staff can begin to efficiently prevent errors, improve patient care and safety, and decrease delays.

http://thejns.org/doi/abs/10.3171/2015.5.JNS142458

KEY WORDS error; cerebrovascular; multidisciplinary; perioperative; surgery; vascular disorders

$\mathrm{U}$ NFILTERED self-reporting of errors was one of the central tenants of the early years of neurological surgery, originating with Harvey Cushing, the founder of modern neurosurgery. ${ }^{14}$ Cushing believed all physicians had the moral obligation to warn others of their errors to prevent their recurrence and that one of the best tools to learn and improve the nascent subspecialty of neurosurgery was to learn from mistakes. However, Cushing and his contemporaries also experienced the backlash of reporting errors. Publications detailing preventable surgical errors temporarily engendered distrust in the subspecialty and put burgeoning neurological surgeons at risk for

ABBREVIATIONS AVM = arteriovenous malformation; $\mathrm{DOC}=$ deviation from an optimal course; $\mathrm{GCS}=$ Glasgow Coma Scale; LOS = length of stay; $\mathrm{mRS}=$ modified Rankin Scale.

SUBMITTED November 23, 2014. ACCEPTED May 7, 2015.

INCLUDE WHEN CITING Published online December 4, 2015; DOI: 10.3171/2015.5.JNS142458. 
legal repercussions, resulting in a decrease in the transparency of self-reporting outside institutional morbidity and mortality conferences that has only recently begun to be overturned.

Since the publication of To Err Is Human by the Institute of Medicine in $1999,{ }^{10}$ there has been a renewed interest in reporting of errors in the medical and surgical communities, yet errors specific to neurosurgery have not been adequately studied. ${ }^{16}$ To Err Is Human concluded that errors were the eighth leading cause of death in the United States, causing almost 100,000 deaths per year. ${ }^{10}$ Other studies have estimated that operative adverse events comprise $48 \%-66 \%$ of all adverse events and that $54 \%-74 \%$ of these are due to preventable errors. ${ }^{2,6,7}$ There is a particular need for error characterization in high-risk subspecialties, such as cerebrovascular surgery, where success is critically dependent on teamwork, advanced technology, and technical skills. A perfect surgery without delays would require flawless communication, infallible equipment, and precise surgical and medical expertise. To work toward that goal, we believe it is imperative to collect and share as much information as possible regarding areas for improvement in both system and individual surgical practices. Furthermore, as a step forward from the days of Cushing, we believe it is just as, if not more, vital to report and analyze near misses (errors that did not result in an adverse event) so we can prevent future errors.

This study improves upon prior research by expanding the scope of information gathering in terms of personnel surveyed and of the type of data elicited. One deficit in previous studies on medical errors was the failure to survey all team members. In all prior studies we could find, only the senior attending surgeon was surveyed, whereas surgery is increasingly a team-based procedure and success is dependent on all players.,17 We hypothesized that by surveying only the senior attending surgeon, previous studies underestimated true error and delay rates and missed errors that may have been observed by other team members (e.g., nurses, anesthesiologists, neurophysiologists).

\section{Methods}

\section{Survey Administration}

Thirty-one cerebrovascular cases performed by the senior author between May and August 2013 were included in a prospective study to identify errors in the operating room. An error was identified as any deviation from an optimal course (DOC), as in prior studies. ${ }^{16,17}$ Immediately following each case, surveys were administered to multiple team members, including the circulating nurse, the anesthesiologist, the resident surgeon, and the attending surgeon. Team members were instructed to report any DOC that had occurred during the surgery, not just the errors attributable or relevant to their role in the surgery. All team members were blinded to the reports of other team members and completed their respective surveys independently.

\section{Error Categorization}

The surveys were organized by operative course (i.e., preoperative, opening, approach, clipping or resection, and closing) to evaluate whether DOCs were more likely to oc- cur in a certain portion of the case; this is particularly important because cerebrovascular cases can be very lengthy depending on the approach and pathology. The portions of the case were defined as follows: preoperative period: the time in the operating room before incision; opening: the portion of the procedure between incision and introduction of the microscope; approach: microscope-assisted dissection of the aneurysm or arteriovenous malformation (AVM) into a clean view; clipping/resection: active aneurysm clipping or AVM resection; closing: all events postclipping/resection until the patient was extubated.

All reported errors were categorized using a modified version of the coding scheme originally developed by Stone and Bernstein. ${ }^{17,19}$ Stone and Bernstein's original classifications included the following: 1) technical errors, 2) contamination, 3) delay, 4) equipment failure or equipment missing, 5) communication, 6) management or judgment, 7) nursing, 8) anesthesia, and 9) other. While, overall, this coding scheme allows for precise categorization, we made 2 modifications. First, we decided that there was no place for an "other" category and that all of the errors reported could be classified into another category. Second, we felt that a delay was never an error in itself but instead was always the result of another error. For example, a microscope malfunction might result in a delay, but the actual error is an equipment failure, not a delay. We felt that categorizing all of the errors that resulted into delays based on their root cause, and keeping track of which original errors caused the most delays, gave more depth and clarity to the findings by highlighting areas for future improvements. Therefore, we used all of Stone and Bernstein's original categories for classification except "other" and "delay." If more than 1 team member reported the same error, it was only counted as a single error, but "duplicate" errors were kept track of and are reported.

\section{Calculated Operating Room Delays}

Scheduled total operating room time and actual operating room time were determined from patient records. We defined the case duration as the time the patient was recorded as entering the operating room until the time the patient left the operating room. Delays were calculated by subtracting the scheduled case duration from the actual case duration.

\section{Outcome Measurements}

To evaluate outcomes, we used the Glasgow Coma Scale (GCS) and modified Rankin Scale (mRS) to score patients' pre- and postoperative neurological statuses and calculated the postoperative length of hospital stay for each patient. The preoperative GCS and $\mathrm{mRS}$ scores were used as a baseline to determine whether any errors reported during a particular case correlated with a net change in the patient's outcome scores. This was done to prevent skewing of the data by postoperative scores that were lower than normal (GCS score of 15 and mRS score of 0 ) but were actually improved from admission.

\section{Analysis}

Statistical analysis was performed using IBM SPSS Statistics software (version 21.0) running on Mac OS X 
10.9 (Apple). Binary correlation analysis was used to determine relationships between reported errors and outcome scores. All averages are presented with a calculated $\mathrm{SD}$. The Student t-test was used to determine significance between 2 averages; significance was defined as $\mathrm{p}<0.05$. Since most prior studies have elicited DOC data from only the attending surgeon, all survey responses recorded by the attending surgeon are parsed out and presented separately.

\section{Institutional Review Board Approval}

This study was conducted under the approval of the University of California, San Francisco, Human Research Protection Program, Committee on Human Research. All patients or their representatives provided informed consent before undergoing surgery.

\section{Results}

\section{Patient/Case Characteristics}

The majority of the cases surveyed were cerebrovascular: $45.2 \%$ were intracranial aneurysm clippings, followed by AVMs (22.6\%) and cavernous malformations (16.1\%) (Table 1). The average patient age was $49.2 \pm 19.8$ years, and $61 \%$ of the patients were female.

\section{Errors: Overview}

For the 31 cases, the team members surveyed (circulating nurse, anesthesiologist, and attending and resident surgeons) reported a total of 118 errors (Table 1). A number of these were duplicates (20.3\%), meaning a net of 94 unique DOCs were captured. The numbers of errors per case ranged from 0 to 8 , with $93.5 \%$ of cases having at least 1 error reported; only 2 of the 31 cases $(6.5 \%)$ had no errors reported (Table 1, Fig. 1). The mean number of errors per case was $3.0 \pm 2.1$.

In comparison, when looking at only the surveys completed by the attending surgeon, 54.8\% of cases were reported as having no DOCs (Table 1). The attending surgeon reported 25 DOCs across 31 cases compared with the 94 reported by all team members, meaning that surveying all team members resulted in the capture of nearly 4 times as many DOCs (or an increase of 276\%) compared with only surveying the attending surgeon. The mean number of errors recorded by the attending surgeon per case was $0.8 \pm 1.2$, which was significantly less than the $3.0 \pm 2.1$ errors per case recorded when all 4 team members were surveyed $(\mathrm{p}<0.001)$.

\section{Types of Errors}

Examples of the types of DOCs reported are detailed in Table 2, and a description of their reported frequencies is detailed in Table 3. Technical errors were the most common DOC reported, constituting nearly one-quarter of all DOCs (24.5\%) (Table 3). Examples of technical errors reported included dural tears, perforator injury, and arterial occlusions detected by indocyanine green. All but 1 of the 23 technical errors recorded were reported by either the attending or resident surgeon. Communication errors were the second most common type of error (22.3\%), and
TABLE 1. Patient and case characteristics

\begin{tabular}{|c|c|c|c|}
\hline Characteristic & $\begin{array}{c}\text { Attending, } \\
\text { Resident, } \\
\text { Anesthesiologist, } \\
\text { Circulating Nurse }\end{array}$ & $\begin{array}{l}\text { Attending } \\
\text { Surgeon } \\
\text { Only }\end{array}$ & $\begin{array}{c}p \\
\text { Value }\end{array}$ \\
\hline \multicolumn{4}{|l|}{ Type of case, no. (\%) } \\
\hline Aneurysm & $14(45.2)$ & - & \\
\hline AVM & $7(22.6)$ & - & \\
\hline Cavernous malformation & $5(16.1)$ & - & \\
\hline Carotid endarterectomy & $1(3.2)$ & - & \\
\hline Other* & $4(12.9)$ & - & \\
\hline \multicolumn{4}{|l|}{ Sex, no. (\%) } \\
\hline M & $12(38.7)$ & - & \\
\hline $\mathrm{F}$ & $19(61.3)$ & - & \\
\hline \multicolumn{4}{|l|}{ Age, yrs } \\
\hline Mean \pm SD & $49.2 \pm 19.8$ & - & \\
\hline Min & 13 & - & \\
\hline Max & 82 & - & \\
\hline \multicolumn{4}{|l|}{ Errors per case, no. } \\
\hline Mean \pm SD & $3.0 \pm 2.1$ & $0.8 \pm 1.2$ & $<0.001$ \\
\hline Min & 0 & 0 & \\
\hline Max & 8 & 5 & \\
\hline Cases w/ $\geq 1$ error, $\%$ & 93.5 & 45.2 & \\
\hline Total errors & 94 & 25 & \\
\hline \multicolumn{4}{|l|}{ Delays per case, no. } \\
\hline Mean \pm SD & $1.2 \pm 1.2$ & $0.03 \pm 0.18$ & $<0.001$ \\
\hline Min & 0 & 0 & \\
\hline $\operatorname{Max}$ & 6 & 1 & \\
\hline Cases $w / \geq 1$ delay, $\%$ & 74.2 & 3.2 & \\
\hline
\end{tabular}

* Bone flap replacement, bypass, cervical laminectomy, dermoid cyst, glioblastoma, hemicraniotomy, and ventriculoperitoneal shunt placement.

the circulating nurse reported the majority of these errors (71.4\%; 15 of 21 reports). Communication errors reported ranged from miscommunication between surgeons (e.g., craniotomy too small) to miscommunication between preoperative and nursing (e.g., preoperative staff did not print surgical consents) and miscommunication between surgeons and nursing (e.g., microscope facing the wrong direction). Management/judgment errors accounted for $16.0 \%$ of reported DOCs, followed by equipment failure or missing equipment DOCs (11.7\%), anesthesia errors (10.6\%), contamination (9.6\%), and nursing (5.3\%).

It is important to compare the "total" column in Table 3 with the "attending" column to evaluate the differences in distribution between the information that would have been collected if only the attending surgeon were surveyed and the error information obtained by surveying all team members. For example, far fewer communication errors would have been captured if only the attending surgeon were surveyed $(22.3 \%$ of total DOCs vs $8.0 \%$ of attending surgeons reported DOCs). Furthermore, greater than $72 \%$ of equipment failure or missing equipment DOCs would have been missed, $90 \%$ of anesthesia errors would have been missed, and no nursing DOCs would have been recorded. 


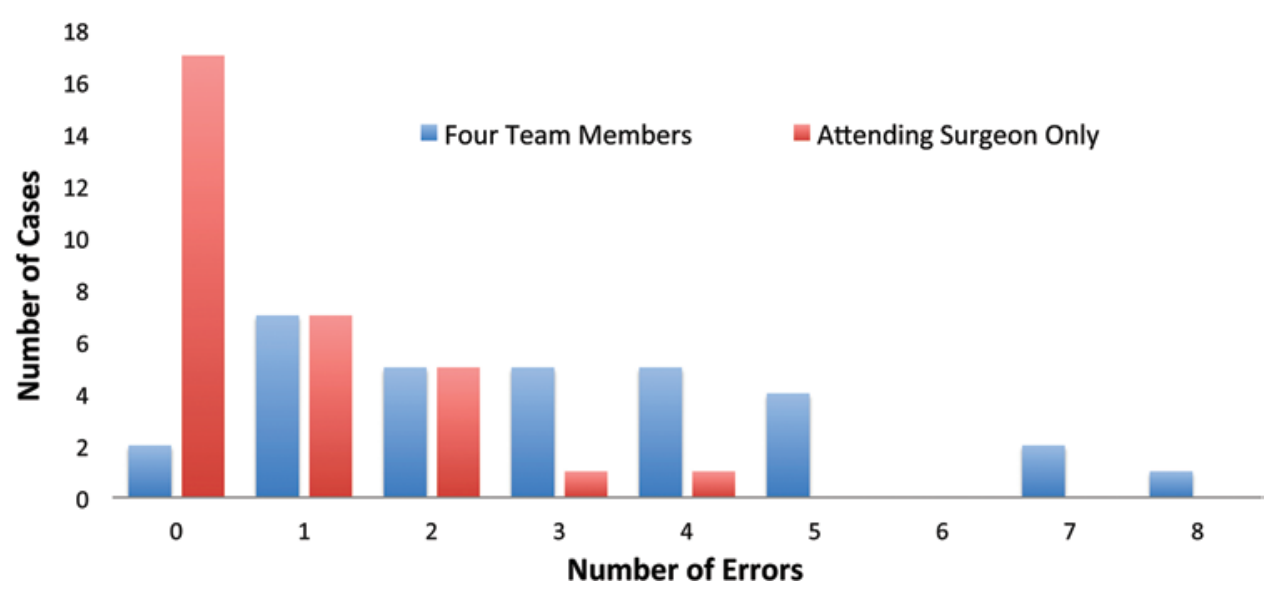

FIG. 1. Number of errors reported per case. Figure is available in color online only.

\section{Reporter Characteristics}

We examined which team members were more likely to report errors, the types of errors they commonly reported, and when they reported them (Table 3, Fig. 2). Resident surgeons reported the most errors (49 of the 94 total; $52.1 \%$ ), followed by the circulating nurses (31.9\%), the attending surgeon (26.6\%), and, last, anesthesiologists (14.9\%). The attending surgeon reported primarily technical (52.0\%) and management/judgment errors (20.0\%), and he was most likely to report DOCs that occurred during clipping/resection. Similar to the attending surgeon, resident surgeons were most likely to report technical errors $(30.6 \%)$, but they also frequently reported other error types as well (management/judgment: $22.4 \%$, equipment failure or missing equipment: 16.3\%; anesthesia: 10.2\%; contamination: $10.2 \%$ ) and reported DOCs occurring across all portions of the procedure. The circulating nurses were second most likely to report DOCs, after the resident surgeons, and reported $31.9 \%$ of all DOCs. They most frequently reported communication errors $(50.0 \%$; 15 errors) and equipment failure or missing equipment (20.0\%; 6 errors). The majority of the DOCs reported by the circulating nurses occurred during the preoperative period. The anesthesiologists reported the fewest errors
(14.9\% of all reports), and when they did report DOCs, they primarily recorded problems with anesthesia (35.7\%; 5 errors) and communication (28.6\%; 4 errors). Like the circulating nurses, the majority of the errors reported by anesthesiologists occurred during the preoperative period.

\section{Perioperative Error Temporality}

The majority of DOCs occurred during the preoperative period (28 errors; $29.8 \%)$ and clipping or resection $(20$ errors; 21.3\%) (Table 4). Communication errors, which included missing consent notes and incorrect setup, were most likely to occur during the preoperative period (15 errors; $71.4 \%$ ), while equipment failure or missing equipment was most likely to occur during the approach (8 errors; $72.7 \%$ ) and technical errors were most common during clipping or resection (11 errors; 47.8\%) and opening $(26.0 \%)$. Note that if only the attending surgeon had been surveyed, the data would have concluded that the majority of DOCs occur during clipping/resection (Fig. 2).

\section{Duplicate Reporting}

It is important to note which errors were "duplicates," or reported individually by more than 1 team members within

\section{TABLE 2. Examples of errors reported}

\begin{tabular}{ll}
\hline \multicolumn{1}{c}{ Error Type } & Examples \\
\hline Technical & "dural tear;" "perforator injury;" "occlusion detected by ICG, corrected" \\
\hline Management/judgment & $\begin{array}{c}\text { "should have planned a larger incision;" "no angiogram available, CTA only;" "better clip configurations;" "needed cervical } \\
\text { carotid exposure, unexpected" }\end{array}$ \\
$\begin{array}{l}\text { Equipment failure or } \\
\text { missing equipment }\end{array}$ & "zoom function on pedal stopped working;" "balancing error;" "suction stopped working, delayed case" \\
\hline $\begin{array}{l}\text { Communication } \\
\text { Anesthesia }\end{array}$ & "not aware of lateral approach until noted on patient's consent form; slight delay in gathering supplies;" "preop did not print \\
Contamination & "difficulty obtaining A-line: 1.5-hr delay;" "patient near wake up during opening, moving in pins" \\
\hline Nursing & "extra incision for hemicraniotomy where it was not prepped;" "dropped bipolars;" "neurophysiology wanted to correct leads" \\
\hline
\end{tabular}

\footnotetext{
* CTA = CT angiography; ICG = indocyanine green
} 
TABLE 3. Types of error reported by each team member and analysis of duplicate errors

\begin{tabular}{|c|c|c|c|c|c|c|}
\hline \multirow[b]{2}{*}{ Error Type } & \multicolumn{6}{|c|}{ No. of Team Members (\%) } \\
\hline & Total & Attending Surgeon & Resident Surgeon & Anesthesiologist & Circulating Nurse & Duplicates \\
\hline Technical & $23(24.5)$ & $13(52.0)$ & $15(30.6)$ & 0 & $1(3.3)$ & $6(20.7)$ \\
\hline Management/judgment & $15(16.0)$ & $5(20.0)$ & $11(22.4)$ & $1(7.1)$ & 0 & $2(11.8)$ \\
\hline Equipment failure or missing & $11(11.7)$ & $2(8.0)$ & $8(16.3)$ & $2(14.3)$ & $6(20.0)$ & $7(38.9)$ \\
\hline Communication & $21(22.3)$ & $2(8.0)$ & $2(4.1)$ & $4(28.6)$ & $15(50.0)$ & $2(8.7)$ \\
\hline Anesthesia & $10(10.6)$ & $1(4.0)$ & $5(10.2)$ & $5(35.7)$ & $3(10.0)$ & $4(28.6)$ \\
\hline Contamination & $9(9.6)$ & $2(8.0)$ & $5(10.2)$ & $1(7.1)$ & $4(13.3)$ & $3(25.0)$ \\
\hline Nursing & $5(5.3)$ & $0(0.0)$ & $3(6.1)$ & $1(7.1)$ & $1(13.3)$ & 0 \\
\hline Total & $94(100)$ & $25(26.6)$ & $49(52.1)$ & $14(14.9)$ & $30(31.9)$ & $24(20.3)$ \\
\hline
\end{tabular}

a case (Table 2). Equipment failure or missing equipment was the most likely DOC to be noted by more than 1 team member (38.9\% overlap in reporting). This means, for example, that more than one-third of the time, if 1 member of the team wrote down "scope malfunctioning," there was a $38.9 \%$ chance another team member would mention on their survey that the microscope malfunctioned during that case. Technical errors, contamination, and problems with anesthesia were also more likely than other DOCs to be reported by more than 1 team member. In particular, when the attending surgeon recorded that a technical deviation from optimal course occurred, the resident also recorded the same technical error $20.7 \%$ of the time.

\section{Error Temporality: Time and Day}

The day of the week of surgery did not have a significant effect on the number of errors per case (Table 5). The start time of the surgery showed a trend that cases with afternoon starts experienced more errors; however, this difference was not significant.

\section{Delays}

More than one-third of the reported DOCs resulted in a delay (40.4\%), with 23 of the 31 cases (74.2\%) reported as experiencing a delay (Table 1). Using patients' operative records, we calculated the actual amount of time surgeries extended past their scheduled operating room time and found a significant positive correlation with the number of delays reported $\left(\chi^{2}=101.4, p=0.028\right)$ (Fig. 3). Compared with cases with no delays reported, cases with 1 delay reported were an average of 5 minutes delayed, cases with 2 delays reported were delayed an average of 18 minutes, and cases with 3 delays reported were on average $64 \mathrm{~min}$ utes delayed.

Delays were by far the most common during the preoperative period (57.1\%), but they occurred during each segment of a surgery (Table 6). Communication, equipment failure or missing equipment, anesthesia, and nursing DOCs were most likely to result in delays: 15 of 21 communication DOCs resulted in a delay, as did 7 of 11 equipment DOCs, 6 of 10 anesthesia DOCs, and 5 of 5 nursing DOCs (Fig. 4). Together, these accounted for the majority of delays (communication: $42.9 \%$; equipment: 20.0\%; anesthesia: $17.1 \%$; and nursing: $14.3 \%$ ). Communication and anesthesia errors resulting in delays were most likely to occur during the preoperative period, while equipment was mostly likely to fail or be found missing during the approach.

Circulating nurses reported the majority of delays, and 25 of the 30 DOCs reported by a circulating nurse resulted in delay (83.3\%) (Fig. 5). The majority of DOCs report-

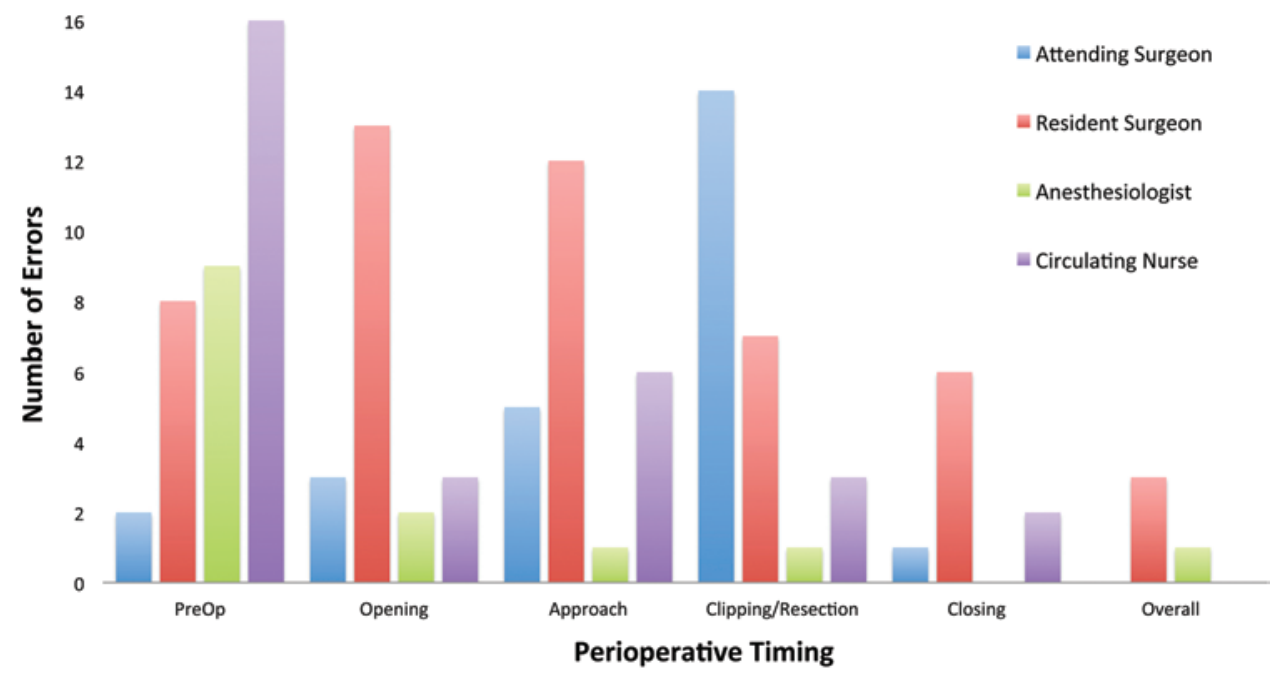

FIG. 2. Perioperative timing of errors, sorted by reporting team member. Figure is available in color online only. 
TABLE 4. Breakdown of errors by category and perioperative timing

\begin{tabular}{lccccccc}
\hline & \multicolumn{9}{c}{ Error Type } & Preop & Opening & Approach & Clipping/Resection & Closing & Overall & $\begin{array}{c}\text { Total No. of Errors } \\
\text { by Type }\end{array}$ \\
\cline { 2 - 7 } Anesthesia & 6 & 2 & 0 & 1 & 1 & 0 & $10(10.6)$ \\
\hline Communication & 15 & 1 & 2 & 1 & 1 & 1 & $21(22.3)$ \\
\hline Contamination & 1 & 3 & 3 & 0 & 2 & 0 & $9(9.6)$ \\
\hline Equipment failure or missing & 2 & 0 & 8 & 1 & 0 & 0 & $11(11.7)$ \\
\hline Management/judgment & 3 & 4 & 1 & 4 & 0 & 3 & $15(16.0)$ \\
\hline Nursing & 1 & 1 & 0 & 2 & 1 & 0 & $5(5.3)$ \\
\hline Technical & 0 & 6 & 3 & 11 & 3 & 0 & $23(24.5)$ \\
\hline Total no. of errors by period & $28(29.8)$ & $17(18.1)$ & $17(18.1)$ & $20(21.3)$ & $8(8.5)$ & $4(4.3)$ & $94(100)$ \\
\hline
\end{tabular}

ed by anesthesiologists also resulted in a delay (10 of 14; $71.4 \%$ ). In contrast, only 12 of the 37 DOCs reported by resident surgeons resulted in a delay $(24.5 \%)$, and only 1 of the 25 DOCs reported by the attending surgeon resulted in a delay (3.2\%).

\section{Outcome Scores}

We conducted a retrospective chart review to determine each patient's long-term neurological outcome using GCS and mRS scores from their most recent follow-up visit and found no significant correlation between reported DOCs and net change in preoperative to postoperative GCS and mRS scores. The median follow-up time was $155 \pm 33$ days.

\section{Length of Stay}

There was a significant correlation between the incidence of technical DOCs and increased length of stay (LOS; $p=0.001$ ) (Fig. 6). The average LOS for a case with no technical errors was $4.4 \pm 0.8$ days; for 1 technical error, $8.7 \pm 2.3$ days; and 2 or more technical errors, $16.2 \pm$ 2.7 days. No other category of DOCs resulted in an increased LOS.

\section{Discussion}

In this prospective study including input from multiple members of the operating team, we found that DOCs

TABLE 5. Temporal error trends*

\begin{tabular}{ccc}
\hline Time & No. of Ops & No. of Errors $\dagger$ \\
\hline Day of week & & \\
\hline Monday & 6 & $1.9 \pm 2.9$ \\
\hline Tuesday & 12 & $2.8 \pm 2.1$ \\
\hline Wednesday & 3 & $2.0 \pm 1.0$ \\
\hline Friday & 10 & $3.6 \pm 1.7$ \\
\hline Saturday & 0 & - \\
\hline Time of day & & $3.0 \pm 2.2$ \\
\hline AM & 22 & $3.6 \pm 1.7$ \\
\hline PM & 9 &
\end{tabular}

* Thirty-one surveys completed by attending surgeon, resident surgeon, anesthesiologist, and circulating nurse.

$\dagger$ Values are presented as the mean \pm SD . occurred in almost all surgeries surveyed and that these DOCs often result in delays and an extended hospital stay. While we did not find any significant correlations between reported DOCs and patients' neurological outcomes scores, a close examination of the reported DOCs reveals many near misses. To our knowledge, this study is the first of its kind to independently survey several members of the operating team (nurses, anesthesiologists, and surgeons) to gather information about medical and surgical errors. This stratification afforded us the opportunity to analyze not only the type of DOCs that occur in a neurosurgical operating room but also which DOCs different team members remembered and chose to report. This role-specific information is critical in formulating feasible interventions to mitigate errors and improve teamwork in the operating room.

\section{Error Categorization and Differential Reporting}

Compiling surveys completed for each case by the circulating nurse, anesthesiologist, resident surgeon, and attending surgeon, we found that errors occurred in $93.5 \%$ of cases, with a mean number of errors per case of $3.0 \pm$ 2.1. Previous studies surveying only the attending operating surgeon have found error rates ranging from $11 \%$ to $87 \% .^{1,17,18}$ In our study, had we only surveyed the attending surgeon, we would have missed nearly $75 \%$ of errors and would have concluded that more than one-half of the surgeries $(54.8 \%)$ were error free. This underscores our hypothesis that previous rates of surgical error likely underestimated their true incidence.

As for the most common type of errors, previous reports using only data collected from the attending surgeons concluded that technical errors were the most common type of error, followed by contamination and equipment failure or missing equipment errors. ${ }^{1,15,17}$ If we only look at the errors reported by the attending surgeon in our study, our breakdown is similar: technical errors were the most common $(52.0 \%)$ followed by management/judgment errors (20.0\%), contamination (8.0\%), equipment failure or missing errors $(8.0 \%)$, and communication $(8.0 \%)$. However, the distribution of errors collected using all team member surveys shows key differences: Technical errors were still found to be most common (24.5\%), but communication errors were far more prevalent $(22.3 \%)$, as were equipment failures (11.7\%) and problems with anesthesia (10.6\%).

Interestingly, the data show a relative paucity of dupli- 


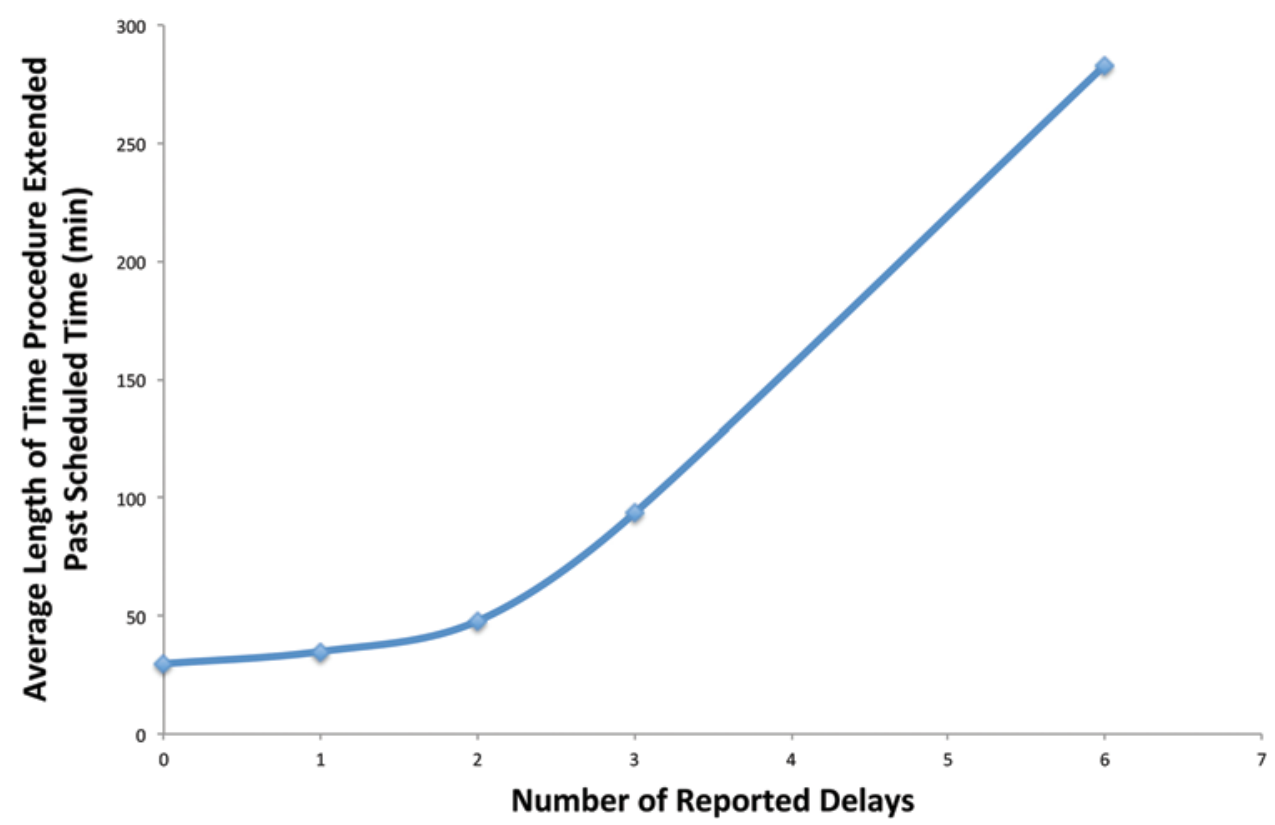

FIG. 3. Average length of time procedure extended past scheduled operating room time versus number of reported delays $\left(\chi^{2}=\right.$ 101.39; $p=0.028$ ). Figure is available in color online only.

cate error reports (only $20.3 \%$ of reports overlapped). Prior to this study, we hypothesized that salient events such as the intraoperative rupture of an aneurysm or a microscope malfunction causing a long delay would be reported by all team members. We also suspected that each team member would contribute equally to the number of overall reports (e.g., each member would report about $25 \%$ of total errors). Instead, we saw that team members were most likely to report only DOCs directly related to their role in the operation and ignore any other errors that occurred outside their domain, even though they were specifically instructed to record all DOCs that occurred during the surgery. This trend occurred across the board: Attending and resident surgeons reported primarily technical $(52.0 \%$ and $30.6 \%$, respectively) and management/judgment errors $(20.0 \%$ and $22.4 \%$, respectively), circulating nurses reported primarily problems with equipment $(20.0 \%)$ or communication errors $(50.0 \%)$, and anesthesiologists reported mostly anesthesia (35.7\%) and communication errors (28.6\%).

These biased reporting trends illustrate the role that individual team members see themselves playing and give us hints on how to improve teamwork and quality of care, and, ultimately, reduce the number of errors. Our results confirmed that surgeons are primarily concerned with DOCs in the surgical aspects of the operation-technique, plan, and equipment. In addition, they reported the majority of errors as having occurred during the opening, approach, and clipping/resection, which are the intensely surgical portions of the operation. In contrast, the circulating nurses and anesthesiologists reported that most errors occurred during the preoperative period, when they are the busiest. The circulating nurses' responses also accurately reflect the role they play in the operating room-the circulating nurse is often said to "run the OR," which includes coordinating multiple team members (leading them to notice more errors in communication $-50.0 \%$ of their reported errors) and ensuring other team members have what they need to perform their duties (leading them to notice and have to correct errors like equipment failure or missing equipment $-20.0 \%$ of their reported errors). While one might hope that their surgeon is primarily preoccupied with surgical aspects of the procedure dur-

TABLE 6. Delays categorized by deviation of optimal course that resulted in the delay and delineated by perioperative period*

\begin{tabular}{lcccccc}
\hline \multicolumn{1}{c}{ Cause of Delay } & Preop & Opening & Approach & Clipping/Resection & Closing & Total Delays by Type, No. (\%) \\
\hline Anesthesia & 6 & 0 & 0 & 0 & 0 & $6(17.1)$ \\
\hline Communication & 10 & 1 & 2 & 1 & 1 & $15(42.9)$ \\
\hline Contamination & 1 & 0 & 0 & 0 & 1 & $2(5.7)$ \\
\hline Equipment failure or missing & 2 & 0 & 4 & 0 & 0 \\
\hline Management/judgment & 0 & 0 & 0 & 0 & 1 & 0 \\
\hline Nursing & 1 & 1 & 0 & 2 & 0 & $5(14.3)$ \\
\hline Technical & 0 & 0 & 0 & 0 & $3(8.6)$ & $35(100)$ \\
\hline Total delays by period, no. (\%) & $20(57.1)$ & $2(5.7)$ & $6(17.1)$ & $4(11.4)$ & & 0 \\
\hline
\end{tabular}

* Data given as no. unless otherwise indicated. 


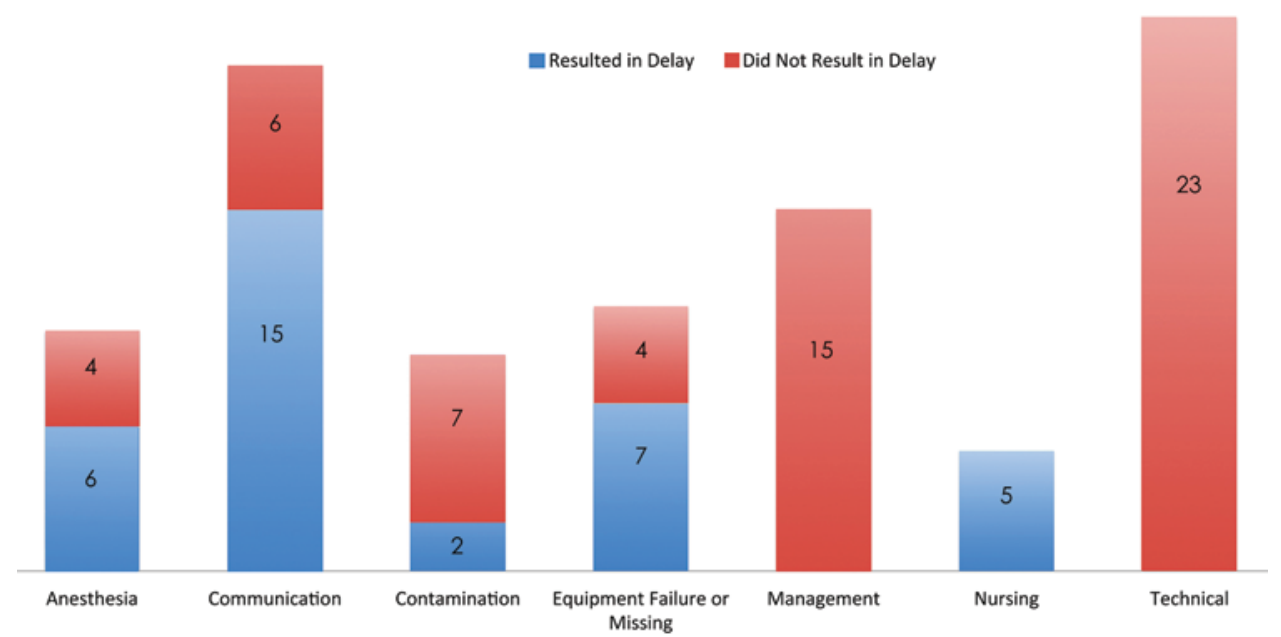

FIG. 4. Percentage of errors that resulted in a delay, sorted by type of error. Figure is available in color online only.

ing the most critical operative portions, when it comes to constructing an accurate, global picture of DOCs in the operating room, their responses leave many gaps. For example, in this study, if we had only surveyed the attending surgeon, as in prior studies, we would have missed $72 \%$ of equipment failure and missing equipment errors, $90 \%$ of anesthesia and communication errors, and $100 \%$ of nursing errors. While it may be expected and considered normal that each team member cares the most about complications and adverse events directly pertinent to their immediate role in the operation, we were still surprised at the blind eye turned to major errors that occurred intraoperatively. The best example of this phenomenon comes from the survey responses of the anesthesiologists. Anesthesiologists reported the fewest errors - only contributing $14.9 \%$ of all errors reported-and neglected to report any technical errors, which were, overall, the most common type of error reported. We found it surprising that even when a particularly salient event occurred, such as aneurysm rupture during clipping, the anesthesiologist failed to

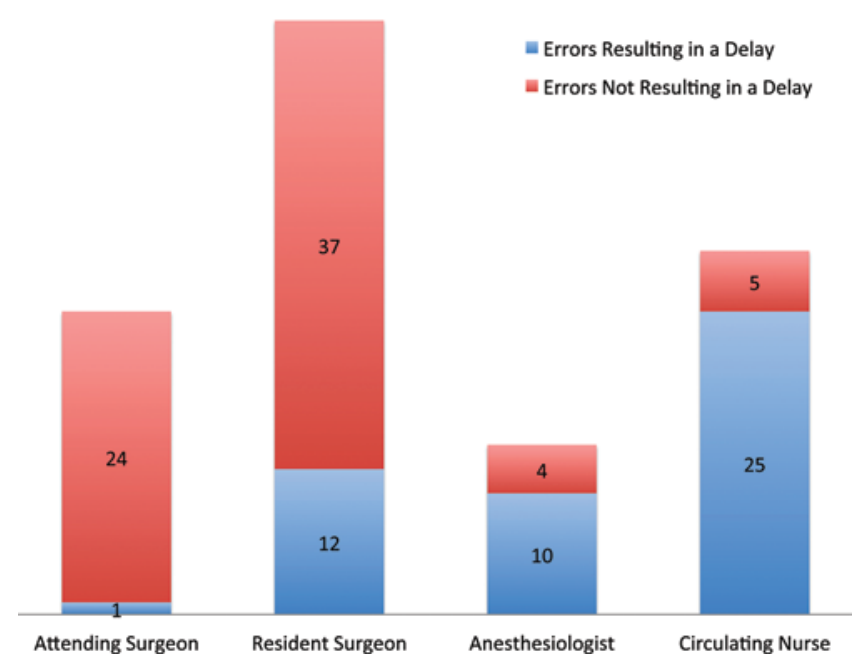

FIG. 5. Delay-related reports as portion of total reports, sorted by reporting team member. Figure is available in color online only. check the box on the survey next to "aneurysm rupture." So, we were forced to ask ourselves, could it be that the anesthesiologists did not realize that the aneurysm ruptured during the surgery, or was it just that they did not think this event was related to their role in surgery and thus did not check it off as a DOC? Either answer would point to a lapse in communication and coordination between the surgeons and anesthesiologists.

Successful neurosurgical outcomes hinge on the coordination of multiple team members. The results of our surveys show a profound lack of awareness of errors as they occur outside one's circumscribed role within the operating room. Teamwork has been repeatedly linked to improved patient outcomes, so addressing these issues is crucial for improving patient safety.

\section{Near Misses and Outcome Scores}

Several studies have shown that participants often rely on error outcomes before deciding whether to report the error, meaning that "near misses" are often not reported. 9 " A major strength of this study is that participants filled out the surveys immediately following the operation and were strongly encouraged to report any deviation from optimal course regardless of their role in it or the outcome of the DOC. By emphasizing these specific instructions and surveying multiple team members, it is our belief that we were able to capture a broader range and severity of DOCs, including many near misses, than prior studies. However, this also means that the majority of recorded DOCs did not result in measureable adverse events. This is underscored by the fact that none of the errors recorded in the surveys were officially reported to the hospital patient safety committee. In addition, the majority of the cases surveyed were scheduled, elective cases with neurologically intact patients (preoperative GCS Score 15, mRS Score $0-1)$. Therefore, it was not surprising to us that we found no significant correlations between DOCs and long-term patient neurological outcome scores on GCS and mRS.

However, we do not feel that the lack of a correlation between DOCs and patient outcomes in any way diminishes the importance of the results presented in this paper. 


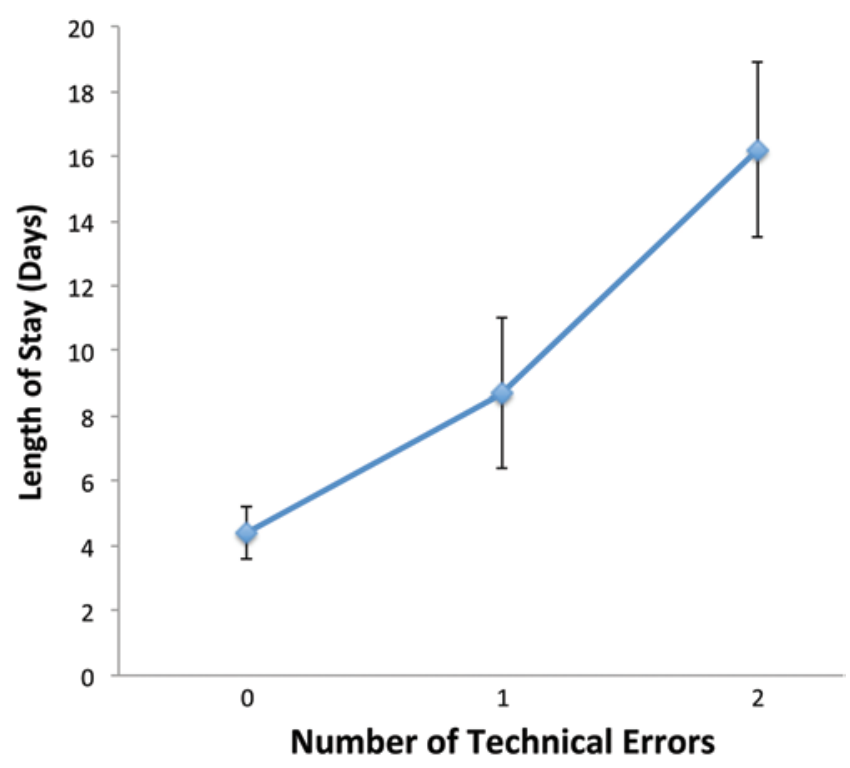

FIG. 6. Number of technical errors and length of postoperative hospital stay $(p=0.001)$. Figure is available in color online only.

In addition to showing that gathering information from multiple members of the operating team results in a vastly more complete picture of areas where we can improve efficiency and areas of potential concern, we successfully identified an array of near misses that could have had potentially catastrophic consequences had it been a different case or an error of slightly greater magnitude. For example, we had 3 different cases where the patient began waking up while he or she was still in Mayfield pins. Fortunately, none of these cases suffered deficits that would be captured by mRS or GCS scores, but there was a real potential for patient harm. Other examples of near misses include the following: 1) a scrub technician opening the wrong stitch, which prolonged temporary clipping time by over 2 minutes during a bypass procedure; 2 ) the need for additional aneurysm clips, which were not available; 3 ) inappropriate and insufficient replacement of blood loss by an anesthesiology resident; 4) a significant delay in mapping equipment setup that led to mapping conducted only after resection; and 5) a malfunctioning operating room chair with an arm rest that abruptly slipped during a noncritical portion of the case.

Importantly, if we have only surveyed the attending surgeon, many of these near misses would have not been reported. This underlines the fact that we cannot rely on the reports of a single member of the operating team to paint a complete picture of errors that occur in the operating room.

\section{Financial Implications of Delays}

Preventing operating room DOCs is a crucial component of decreasing perioperative delays, which have broad implications for the financial sustainability of our health care system. We found that $74.2 \%$ of cases experienced at least 1 error that resulted in a delay and, on average, there were 1.23 delays reported per case. Both of these metrics were higher than prior estimates. A 2010 study by Wong et al. estimated that delays occur in $51.4 \%$ of cases with an average of 0.77 delays per case. ${ }^{19}$ Another study by Overdyk et al. found 0.63 delays per case.

Estimating the precise cost per minute is notoriously difficult and dependent upon many factors. ${ }^{13}$ It necessitates taking into account the cost of the time of numerous personnel involved in a cerebrovascular case (attending surgeon, resident surgeon, anesthesiologist, circulating nurse, scrub technician, neurophysiologist, equipment technician), the cost per minute of the operating room itself, and the opportunity costs of other uses for the personnel, operating room, and equipment.

Previous studies have estimated the cost of an operating room to range from $\$ 2.81$ to $\$ 15.57$ per minute..$^{3,4,12}$ Cleveland Clinic, in accordance with Ohio law, reports its operating room costs for a high-complexity case, such as an intracranial procedure (Level 6), as \$3678 for each additional 30 minutes, leading to a cost of $\$ 122.60$ per minute, not including equipment, anesthesia, or surgeon fees. ${ }^{5}$

In our study, we found that subjectively reported delays were significantly correlated with actual delays, such that 1 reported delay corresponded to an average actual operating room delay of 5 minutes, 2 reported delays corresponded to an 18-minute delay, and 3 delays corresponded to an average of a 64-minute delay. This suggests that delays compound exponentially, not linearly. Using the lowest reported cost per minute, this would result in an average additional cost of $\$ 14.05$ for 1 reported delay, $\$ 50.58$ for 2 delays, and $\$ 179.84$ for 3 delays. Using the highest estimate, which likely more accurately reflects billing for cerebrovascular cases, 1 delay costs $\$ 613.00,2$ delays $\$ 2206.80$, and 3 delays $\$ 7846.40$. In our study, with 31 cases and an average of 1.23 delays per case ( 8 minutes), this results in an estimated preventable cost of between $\$ 22.48$ and $\$ 980.80$ per case and $\$ 696.88$ to $\$ 30,404.80$ for the study period.

\section{Root Causes of Operating Room Delays}

Our study is unique in that we have access to the root cause of reported delays and to their perioperative timing, allowing us to parse out areas for intervention (Table 6, Fig. 4). Nurses and anesthesiologists were most likely to report errors that resulted in delays: 25 of the 30 errors reported by the nursing staff resulted in a delay (83.3\%) and 10 of the 14 errors reported by anesthesiologists resulted in a delay $(71.4 \%)$. In comparison, very few of the errors reported by the attending ( 1 of $25 ; 4.0 \%$ ) and resident surgeons $(12$ of $49 ; 24.5 \%)$ resulted in delays. If only the attending surgeon had been surveyed, a great deal of data about the true incidence of delays in the operating room would have been lost, and we would have estimated that only $3.2 \%$ of cases experienced a delay. This is particularly important given the high financial consequences of delays in the operating room.

Most delays (57.1\%) occurred during the preoperative period and were due to communication or anesthesia errors. Equipment failure during the approach was also a common cause of delay. Similarly, prior studies have shown equipment failure or unavailability and anesthesia problems were the most common causes of delays..$^{8,19}$ However, these studies failed to show the significant role of communication errors in perioperative delays, likely 
because the majority of communication errors (19 of 21 errors) were reported by staff not surveyed in prior studies (e.g., circulating nurse and anesthesiologist).

Given that multiple, long delays were caused by difficulty with anesthesia in the preoperative period, a potential intervention may be to alert senior anesthesiologists when a patient is ready for intubation so that they are on call if the resident anesthesiologist for the case is having difficulty inserting an arterial line or intubating a patient. Along similar lines, equipment failure or missing equipment almost always required that the circulating nurse call a technician to come investigate the problem. The frequent unavailability of a technician produced further delays. Since equipment failures causing delays almost always occurred during the transition to microscope approach (at least in this study), it could be worthwhile to alert the technicians a few minutes before the approach begins to ask them to be on standby in case equipment malfunctions, or to find better ways to test and investigate the microscope before the case begins. With equipment malfunctions causing delays in nearly $20 \%$ of cases, this would likely be a cost-effective intervention.

\section{Length of Stay}

Our analysis showed that technical DOCs were the only type of reported errors significantly correlated with an increased LOS. Similar to our data presented on delays, the average LOS increased exponentially with more reported technical errors, rather than linearly. A patient whose case had no technical errors experienced an average LOS of $4.4 \pm 0.8$ days; 1 technical error, $8.7 \pm 2.3$ days, 2 or more technical errors, $16.2 \pm 2.7$ days. Technical errors occurred in $48.4 \%$ of cases, with an average of $0.74 \pm 0.99$ technical errors per case. The Healthcare Blue Book (http://www. healthcarebluebook.com), a website that publishes nationally competitive prices for health care services, estimates the average cost of a hospital night at $\$ 1800$. In our analysis, this corresponds to an additional incurred cost of approximately $\$ 7200$ for 1 technical error and $\$ 21,600$ for 2 or more technical errors. For the 31 cases in this study, this amounts to an estimated $\$ 180,000(\$ 7200 \times 10$ cases with 1 technical error $+\$ 21,600 \times 5$ cases with 2 or more technical errors).

\section{Strengths and Limitations}

There are several strengths of this study, most prominently the inclusion of survey results from multiple members of the operating team from different disciplines, and the separation of delays as the results of errors rather than errors in themselves.

There are also several limitations of this study. Like any subjective survey, this study relies on team members to remember and accurately report errors. Part of the bias in error reporting likely reflects the salience of errors experienced by each team member. That is, the operating surgeon is more likely to experience a technical error more profoundly than an anesthesia error, which will influence which errors they remember, report, and focus on. While the inclusion of additional team members provided more data that would have been missed if only the attending surgeon had been surveyed, the low amount of duplicate errors suggests that a great deal of errors still went unreported.
Another significant limitation of the study is the limited sample size. Though we were able to capture a large number of errors and correlations between errors and LOS, the limited number of patients reduces this study's power to detect some correlations, like those between the number of errors and patient outcome. Though we did not detect a correlation between errors and outcome here, one might be found with a sufficiently large sample.

\section{Conclusions}

This study demonstrates that error is truly in the eye of the beholder-the types and timing of perioperative errors vary based on whom you ask. While the majority of prior studies have calculated error rates and determined interventions based solely on the reports of the attending surgeon, we found that this method misses nearly $75 \%$ of errors. By compiling reports from all team members, we found a significant correlation between technical deviations from optimal course and short-term patient outcomes (i.e., a prolonged hospital stay). We also found compelling correlations between reported delays and actual operating room delays, which would have almost certainly been missed if only the attending physician had been surveyed. Using the specific root causes of the delays recorded in the surveys, we were able to precisely identify areas where efficiency and teamwork can be improved, which will have potentially profound financial implications by reducing the number of perioperative delays. These results emphasize that independently soliciting information from each staff member in the operating room is essential in developing a complete picture of errors occurring in the operating room. It is only by understanding every perspective that we can begin to efficiently decrease errors, improve patient care and safety, and decrease delays.

\section{References}

1. Boström J, Yacoub A, Schramm J: Prospective collection and analysis of error data in a neurosurgical clinic. Clin Neurol Neurosurg 112:314-319, 2010

2. Brennan TA, Leape LL, Laird NM, Hebert L, Localio AR, Lawthers AG, et al: Incidence of adverse events and negligence in hospitalized patients. Results of the Harvard Medical Practice Study I. N Engl J Med 324:370-376, 1991

3. Bridges M, Diamond DL: The financial impact of teaching surgical residents in the operating room. Am J Surg 177:2832, 1999

4. Chamberlain RS, Patil S, Minja EJ, Kordears K IV: Does residents' involvement in mastectomy cases increase operative cost? If so, who should bear the cost? J Surg Res 178:18-27, 2012

5. Cleveland Clinic: Patient Price Information List. (http:// my.clevelandclinic.org/ccf/media/files/Patients/clevelandclinic-main-charges.pdf) [Accessed August 26, 2015]

6. Etchells E, O'Neill C, Bernstein M: Patient safety in surgery: error detection and prevention. World J Surg 27:936-942, 2003

7. Gawande AA, Thomas EJ, Zinner MJ, Brennan TA: The incidence and nature of surgical adverse events in Colorado and Utah in 1992. Surgery 126:66-75, 1999

8. Harders M, Malangoni MA, Weight S, Sidhu T: Improving operating room efficiency through process redesign. Surgery 140:509-516, 2006

9. Kaldjian LC, Jones EW, Wu BJ, Forman-Hoffman VL, Levi 
BH, Rosenthal GE: Reporting medical errors to improve patient safety: a survey of physicians in teaching hospitals. Arch Intern Med 168:40-46, 2008

10. Kohn LT, Corrigan JM, Donaldson MS: To Err Is Human: Building a Safer Health System. Washington, DC: National Academies Press, 2000, Vol 627

11. Macario A: What does one minute of operating room time cost? J Clin Anesth 22:233-236, 2010

12. Nelson DF, Emerick T, Giedraitis A, Hudson M: An analysis of operating room cost per surgical minute as a measure of efficiency across a large multi-hospital health care system, in American Society of Anesthesiologists Conference on Practice Management. Schaumburg, IL: American Society of Anesthesiologists, 2015 (Abstract PM21)

13. Overdyk FJ, Harvey SC, Fishman RL, et al: Successful strategies for improving operating room efficiency at academic institutions. Anesth Analg 86:896-906, 1998

14. Pinkus RLB: Mistakes as a social construct: an historical approach. Kennedy Inst Ethics J 11:117-133, 2001

15. Regenbogen SE, Greenberg CC, Studdert DM, Lipsitz SR, Zinner MJ, Gawande AA: Patterns of technical error among surgical malpractice claims: an analysis of strategies to prevent injury to surgical patients. Ann Surg 246:705-711, 2007

16. Rolston JD, Zygourakis CC, Han SJ, Lau CY, Berger MS, Parsa AT: Medical errors in neurosurgery. Surg Neurol Int 5 (Suppl 10):S435-S440, 2014

17. Stone $\mathrm{S}$, Bernstein M: Prospective error recording in surgery: an analysis of 1108 elective neurosurgical cases. Neurosurgery 60:1075-1082, 2007
18. Wirbel R, Yacoub A, Dehne M: Prospective collection of error data in orthopaedic and trauma surgery procedures. Int Orthop 38:2369-2375, 2014

19. Wong J, Khu KJ, Kaderali Z, Bernstein M: Delays in the operating room: signs of an imperfect system. Can J Surg 53:189-195, 2010

\section{Disclosures}

Dr. Rolston was supported in part by a socioeconomic fellowship from the Congress of Neurological Surgeons.

\section{Author Contributions}

Conception and design: all authors. Acquisition of data: all authors. Analysis and interpretation of data: all authors. Drafting the article: all authors. Critically revising the article: all authors. Reviewed submitted version of manuscript: all authors. Approved the final version of the manuscript on behalf of all authors: Lawton. Statistical analysis: Michalak. Study supervision: Lawton.

\section{Correspondence}

Michael T. Lawton, MD, Department of Neurological Surgery, University of California, San Francisco, 505 Parnassus Ave., M779, San Francisco, CA 94143-0112.email: LawtonM@ neurosurg.ucsf.edu. 